\title{
Highly Depth-sensitive TEM Imaging of Graphene by using Monochromatic Electron Source at Low Accelerating Voltage
}

\author{
Shigeyuki Morishita ${ }^{1}$, Ryosuke Senga ${ }^{2}$, Yung-Chang Lin ${ }^{2}$, Hidetaka Sawada ${ }^{1}$ and Kazu Suenaga ${ }^{2}$ \\ 1. JEOL Ltd, Akishima, Tokyo, Japan. \\ 2. National Institute of Advanced Industrial Science and Technology (AIST), Tsukuba, Ibaraki, Japan.
}

The spatial resolution of electron microscopy has been already improved to be sub-angstrom and is sufficient for analysis of atomic structure. In addition to the $\mathrm{x}-\mathrm{y}$ specimen plane resolution, we should improve resolution in $\mathrm{z}$ (depth) direction to perform three-dimensional (3D) atomic structure analysis. However, the resolution in $\mathrm{z}$ direction is mostly still at nanometer-order although sub-nanometer depth resolution was realized by a specific post processing technique using a series of defocused images [1]. For the 3D observation, electron tomography is typically used. In these days, through focal imaging or optical sectioning are also used for extracting 3D information from images without specimen tilt. To improve the depth resolution in these methods, focal depth should be reduced. In the case of low-voltage aberration-corrected transmission electron microscopy (TEM), the focal depth is limited by defocus spread due to chromatic aberration and energy spread of an electron source. In this paper, we demonstrate highly depth-sensitive TEM imaging at low voltage with a monochromatic electron source.

First, we simulated TEM images and evaluated the depth sensitivity improvement on energy spread realized by a monochromator. Figure 1a shows simulated TEM images of monolayer graphene at various foci. The peak intensities at an atomic position for various energy spreads are plotted in Fig. $1 \mathrm{~b}$. This graph shows that the contrast of the carbon atom on their foci changes more as the energy spread is smaller. A gradient of this graph shows a focal sensitivity of the image contrast, because the focal sensitivity increases with reducing energy spread, the depth sensitivity would be improved by using a monochromatic source.

Second, we demonstrated highly depth-sensitive imaging by using a sample of bilayer graphene, which was sometimes used for evaluation of the depth sensitivity [1,2]. Figure 2 shows the experimental TEM image of bilayer graphene taken at $60 \mathrm{kV}$. For the observation, we used a TEM with delta-type geometrical aberration correctors [3] in probe and image forming systems and a monochromator [4] to improve lateral resolution [5,6] and depth resolution. The image was obtained at defocus of $-1 \mathrm{~nm}$, and black dots in the image indicate the carbon atoms. The carbon atoms in the bilayer graphene have three different contrasts. The darkest contrast shows two atoms superimposed in z-direction; the middle contrast shows one atom in lower layer; and the brightest contrast shows one atom in upper layer. The difference of these contrasts distinguished the height of the carbons in upper or lower layer. The height difference of two graphene sheets is $0.34 \mathrm{~nm}$. Thus, the image represents the depth resolution better than $0.34 \mathrm{~nm}$. A histogram of this image also shows that precision of height is better than 1 angstrom. We concluded that the monochromator is effective for the highly depth-sensitive TEM imaging.

By using this highly depth-sensitive microscope, we observed monolayer graphene with dislocations. The dislocations are expected to make buckling structure of graphene [7]. Comparing experimental TEM images of graphene with and without dislocations, we detected that a part of carbon atoms between the dislocations are elevated. We can elucidate that this depth variation of monolayer graphene sheet caused by the dislocations. 
In this paper, we obtained 3D information from single images of the bilayer graphene and monolayer graphene dislocations because these specimens have simple structures. For observing more complicated materials, through focal imaging would be required for obtaining more information. Even in this case, the proposed microscope would be effective due to its high depth sensitivity with short focal depth.

\section{References}

[1] D Van Dyke et al, Nature 486 (2012) p. 243.

[2] L Chen et al, Sci. Rep. 7 (2017) p. 10839.

[3] H Sawada et al, J. Electron Microsc. 58 (2009) p. 341.

[4] M Mukai et al, Ultramicroscopy 140 (2014) p. 37.

[5] S Morishita et al, Appl. Phys. Lett. 108 (2016) p. 013107.

[6] S Morishita et al, Phys. Rev. Lett. 117 (2016) p. 153004.

[7] O Lehtinen et al, Nat. Commun. 4 (2013) p. 2098.

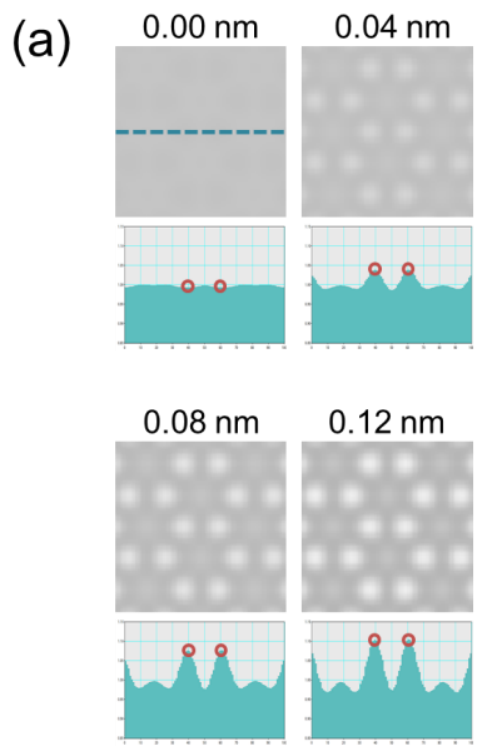

(b)

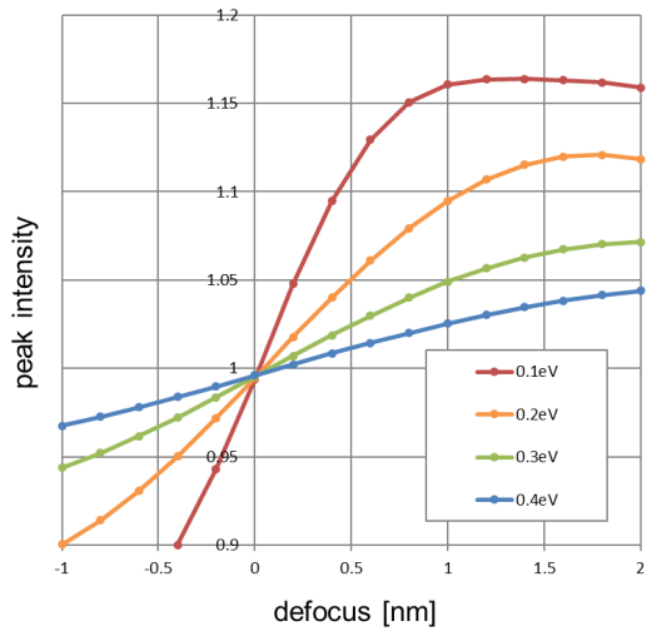

Figure 1. (a) Simulated TEM images of graphene with various defoci and their intensity line profiles. (b) Relationship between peak intensity of carbon images and defocus. The gradient of this graph shows the focal sensitivity.

(a)

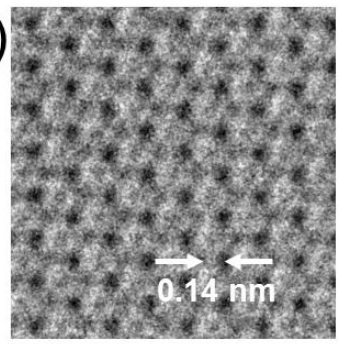

(b)

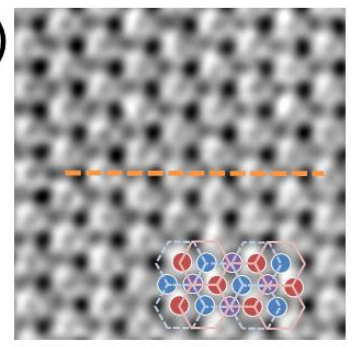

(c)

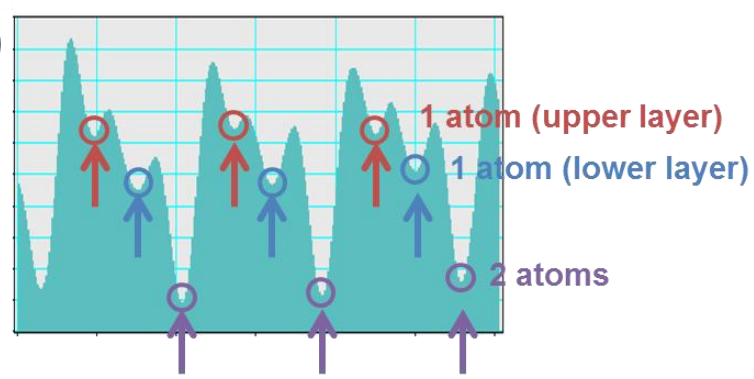

Figure 2. (a) TEM image of bilayer graphene. (b) Low-pass filtered image of (a). (c) Intensity line profile along the dotted line in (b). 